\title{
Correlation between dermal thickness and scar formation in female patients after thyroidectomy
}

\author{
Hong II Kim \\ Chan Yee Kwak', \\ Hyo Young Kim ${ }^{1}$, \\ Hyung Suk $\mathrm{Yi}^{1}$, \\ Eun Ju Park', \\ Jeong Hoon Kim², \\ Jin Hyung Park ${ }^{1}$
}

Departments of ${ }^{1}$ Plastic and Reconstructive Surgery and ${ }^{2}$ Surgery, Kosin University Gospel Hospital, Kosin University College of Medicine, Busan, Korea

\begin{abstract}
Background: Minimizing scarring has long been a challenge in plastic surgery. Factors affecting scar formation are well known, but the effect of some patient-specific factors such as dermal thickness remains unverified. Management of factors predictive of scarring can improve postoperative patient satisfaction and scar treatment. Methods: For 3 years, we used ultrasonography to measure dermal thickness in female patients who had undergone thyroidectomy for cancer at our hospital. We confirmed the influence of dermal thickness on hypertrophic scar formation and the Patient and Observer Scar Assessment Scale scar score 6 months after surgery.

Results: There was a positive correlation between dermal thickness and scar score $(p<0.05)$, and dermal thickness appears to be a cause of hypertrophic scar formation $(p<0.05)$.

Conclusion: Thick dermis was found to cause poor scar formation and hypertrophic scarring. Prediction of factors that can influence scar formation can be used to educate patients before surgery and can help in scar management and improvement in patient satisfaction.
\end{abstract}

Keywords: Skinfold thickness / Hypertrophic cicatrix

\section{INTRODUCTION}

Minimizing of surgical scars is very important in plastic surgery. Identifying factors predicting of hypertrophic scarring or bad aesthetic results can be used to educate patients prior to surgery and possibly increase postoperative satisfaction [1]. Proper suture technique, prevention of wound infections and foreign-body reactions, and wound care are known to affect scar formation $[2,3]$. However, patient-specific factors such as dermal thickness are not well understood. We have observed that patients with thick skin had worse aesthetic results than patients with thinner skin. Several studies have examined whether skin thickness affects scar formation $[4,5]$.

\section{Correspondence: Jin Hyung Park}

Department of Plastic and Reconstructive Surgery, Kosin University College of Medicine, 262 Gamcheon-ro, Seo-gu, Busan 49267, Korea

E-mail: atreyue@naver.com

Received May 16, 2018 / Revised June 18, 2018 / Accepted June 20, 2018
In this study, dermal thickness in patients who underwent thyroidectomy through a transverse anterior cervical incision was investigated. The aim of this study was to determine the relationship between dermal thickness and scar formation through measurement of dermal thickness with preoperative ultrasonography. We hypothesized that thicker dermis may be a risk factor for hypertrophic scarring and poor scar formation.

\section{METHODS}

\section{Study subjects}

This study included 290 female patients aged 19 to 55 years who underwent thyroidectomy through a transverse cervical incision, performed by the same surgeon (J.H.K) between December 2015 and August 2017. After thyroidectomy, platysma muscle suture and skin closure was done by one experienced plastic surgeon (J.H.P). Male patients, and those with a history of thyroid cancer 
surgery at the same site, radiation therapy, local steroid injections, obesity (body mass index $>25 \mathrm{~kg} / \mathrm{m}^{2}$ ), diabetes history, chronic kidney diseases history and an extended surgical procedure were excluded. After surgery, all patients were taken intravenous antibiotics (empirical 3rd generation cephalosporin) and stitches out were performed in postoperative 5 days. After stitched out, to reinforced skin closure, Histoacryl (B. Braun Surgical, Barcelona, Spain) was applied. And after 7 days from Histoacryl applied, surgical tape Steri-Strip (3M, Neuss, Germany) were applied for another 7 days. Thereafter, surgical tape were removed and patients use silicone ointment and silicone gel sheets Bapscarcare (BAP Medical, Apeldoorn, The Netherlands) until 6 months postoperatively.

\section{Preoperative evaluation and measurement of dermal thickness}

Preoperatively, all patients underwent ultrasonography. Dermal thickness was measured at the anterior neck using a 51-Hz, broadband linear ultrasound probe (Philips EPIQ 7G systems, Andover, MA, USA). A probe with a small amount of ultrasonic gel was placed on the anterior neck to evaluate the superior thyroid notch, cricoid cartilage, and isthmus of the thyroid gland. Lateral neck placement was used to evaluate lymph nodes. Measurements were taken before the operation at the same time of day, 1 month before surgery.

The electronic calipers measured the distance from the outer epidermal surface to the underlying dermis, just above the subcutaneous fat layer, on a two-dimensional B-mode image. Because all surgical incisions were performed at the same level, all ultrasound images were assessed at the upper margin of the isthmus of the thyroid gland. This level is two fingerbreadths superior to the jugular notch. The dermal thickness was also measured at the left and right lateral lobes at the level of the central isthmus, and the mean total dermal thickness values were calculated for each patient (Fig. 1).

\section{Postoperative scar assessment}

Clinical photographs were taken preoperatively and 6 months postoperatively. Scar formation was assessed by two expert plastic surgeons (H.I.K and J.H.P) at 6 months postoperatively. Two plastic surgeons performed scar evaluations using the Patient and Observer Scar Assessment Scale (POSAS) at 6 months postoperatively (Fig. 2) [6,7]. Assessment items were vascularity, pigmentation, thickness, relief, pliability, surface area. Each of the six items is scored numerically (range, 6 to 60). The lowest score 6 means normal skin, highest score 60 means the worst scar. Patients with hypertrophic scarring were separately classified. Immature raised,
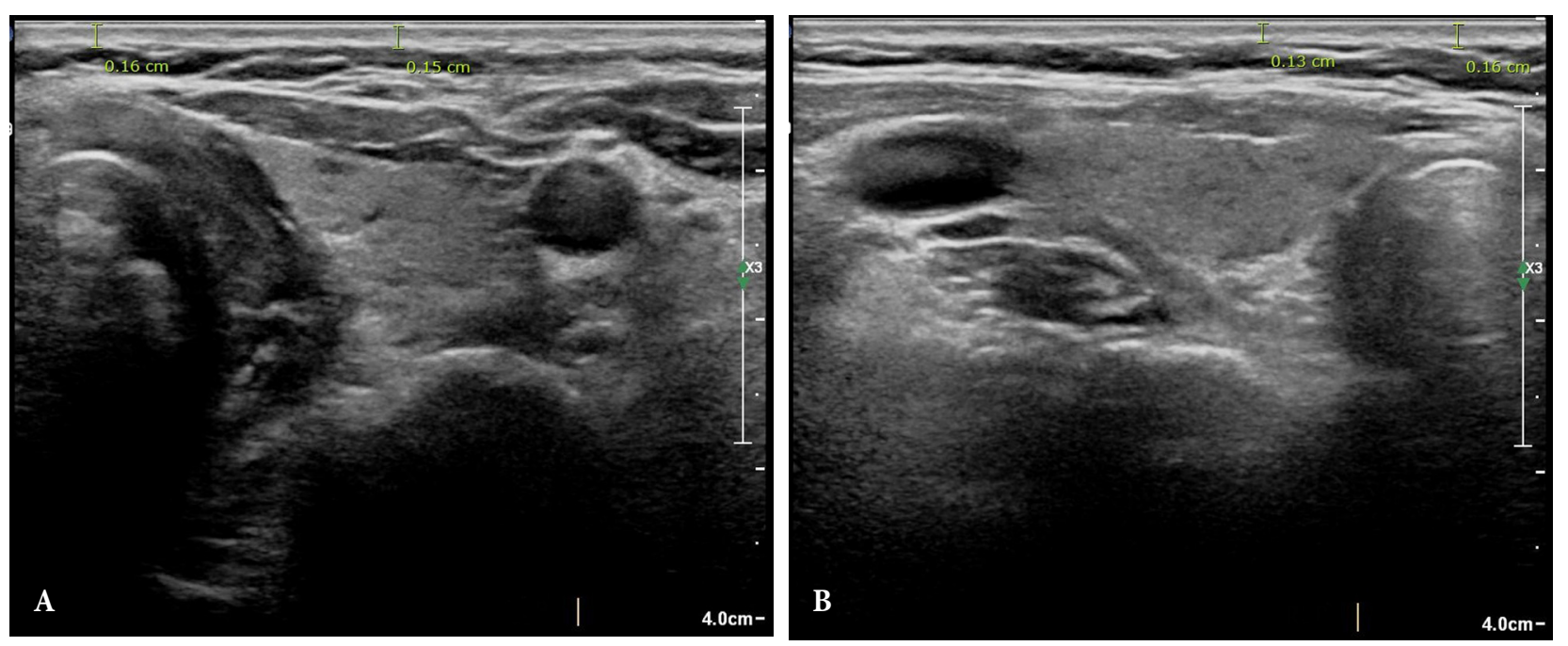

Fig. 1. Preoperative ultrasonography. The distance from the outer epidermal surface to the underlying dermis below the subcutaneous fat layer was determined using two-dimensional B-mode imaging. The average of each distance was measured. Left (A) and right (B) lobe of thyroid at the level of the central isthmus. 
mature raised scar, erythematous raised scar were classified hypertrophic scar. We attached to a skin template to help assessing hypertrophic scars; VIII and IX type scar were typical hypertrophic scars [8].

\section{Statistical analysis}

Statistical analysis was conducted using SPSS ver. 18.0 (SPSS Inc., Chicago, IL, USA). The associations between dermal thickness and scar scores were analyzed using Pearson correlation analysis. Dermal thickness and hypertrophic scars were analyzed using lo-

\begin{tabular}{|c|c|c|c|c|c|c|c|c|c|c|c|c|c|}
\hline & & Normal skin & 1 & 2 & 3 & 4 & 5 & 6 & 7 & 8 & 9 & 10 & $\begin{array}{l}\text { Worst scar } \\
\text { imaginable }\end{array}$ \\
\hline \multirow{5}{*}{ Vascularity } & Pale & & & & & & & & & & & & \\
\hline & Pink & & & & & & & & & & & & \\
\hline & Red & & & & & & & & . & & & & \\
\hline & Purple & & & & & & & & & & & & \\
\hline & Mix & & & & & & & & & & & & \\
\hline \multirow{3}{*}{ Pigmentation } & Нypo & & & & & & & & & & & & \\
\hline & Hyper & & & & & & & & - & & & & \\
\hline & Mix & & & & & & & & - & & & & \\
\hline \multirow{2}{*}{ Thickness } & Thicker & & & & & & & & & & & & \\
\hline & Thinner & & & & & & & & & & & & \\
\hline \multirow{3}{*}{ Relief } & More & & & & & & & & & & & & \\
\hline & Less & & & & & & & & & & & & \\
\hline & Mix & & & & & & & & & & & & \\
\hline \multirow{3}{*}{ Pliability } & Supple & & & & & & & & & & & & \\
\hline & Stiff & & & & & & & & & & & & \\
\hline & Mix & & & & & & & & & & & & \\
\hline \multirow{3}{*}{ Surface area } & Expansion & & & & & & & & & & & & \\
\hline & Contraction & & & & & & & & & & & & \\
\hline & Mix & & & & & & & & & & & & \\
\hline \multicolumn{2}{|c|}{ Overall opinion } & & & & & & & & & & & & \\
\hline
\end{tabular}

Fig. 2. Observer scar scales for evaluation of scar formation. 
gistic regression analysis. In all statistical comparisons, significance was determined by a $p$-value less than 0.05 , respectively.

\section{RESULTS}

The study included 290 female patients with a mean age of $38.43 \pm$ 7.69 years (range, 19 to 55 years). The average dermal thickness was $0.16 \pm 0.03 \mathrm{~mm}$ (range, 0.10 to $0.28 \mathrm{~mm}$ ). At 6 months postoperatively, hypertrophic scarring was observed in 19 cases (6.55\%), There were no keloid scars (Fig. 3). The average total scar score (POSAS, n=290) was 21.74 \pm 7.41 (range, 8 to 43). In hypertrophic scar cases $(n=19)$, the mean scar score was $27.58 \pm 7.34$, and the mean thickness was $0.18 \pm 0.03 \mathrm{~mm}$. When hypertrophic scarring cases were excluded ( $\mathrm{n}=271$ ), the mean scar score was 21.28 \pm 7.41 , with mean thickness $0.16 \pm 0.03 \mathrm{~mm}$ (Table 1). Dermal thickness values were compared with POSAS scores using Pearson correlation analysis. Dermal thickness and scar scores were significantly
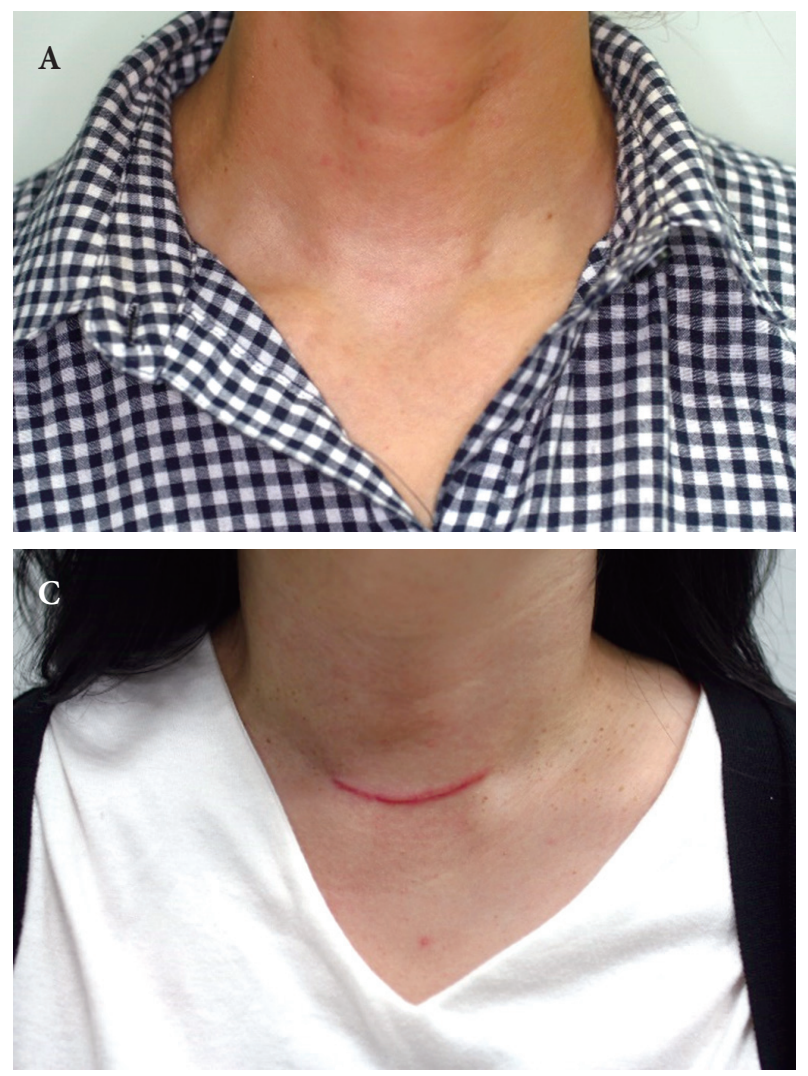

\section{DISCUSSION}

Our hypothesis that thicker dermis may be a risk factor for poor scar development and hypertrophic scarring was supported by the results of this study. Dermal thickness was a risk factor for

Fig. 3. Four different scars are presented in anterior neck clinical photographs. (A) Fine line scar. (B) Stretched scar. (C) Hypertrophic scar. (D) Depressed scar. 
Table 1. Mean scar scores and thickness according to hypertrophic scar, excluding hypertrophic scar cases

\begin{tabular}{|c|c|c|c|c|}
\hline Variable & $\begin{array}{l}\text { Hypertrophic scar case } \\
(n=19)\end{array}$ & $\begin{array}{l}\text { Excluding hypertrophic } \\
\text { scar case }(n=271)\end{array}$ & $p$-value & Odds ratio \\
\hline Age (yr) & $38.78 \pm 7.59$ & $38.44 \pm 7.70$ & 0.967 & 0.999 \\
\hline POSAS score & $27.58 \pm 7.34$ & $21.28 \pm 7.41$ & 0.097 & 1.064 \\
\hline Dermal thickness (mm) & $0.18 \pm 0.03$ & $0.16 \pm 0.03$ & $0.001^{*}$ & $1.343^{\mathrm{a})}$ \\
\hline
\end{tabular}

Values are presented as mean \pm standard deviation.

POSAS, Patient and Observer Scar Assessment Scale.

a) Odds ratio by logistic regression analysis: for each increase in thickness by $0.1 \mathrm{~mm}$, hypertrophic scar formation is increased by an odds ratio=1.343. ${ }^{*} p<0.05$.

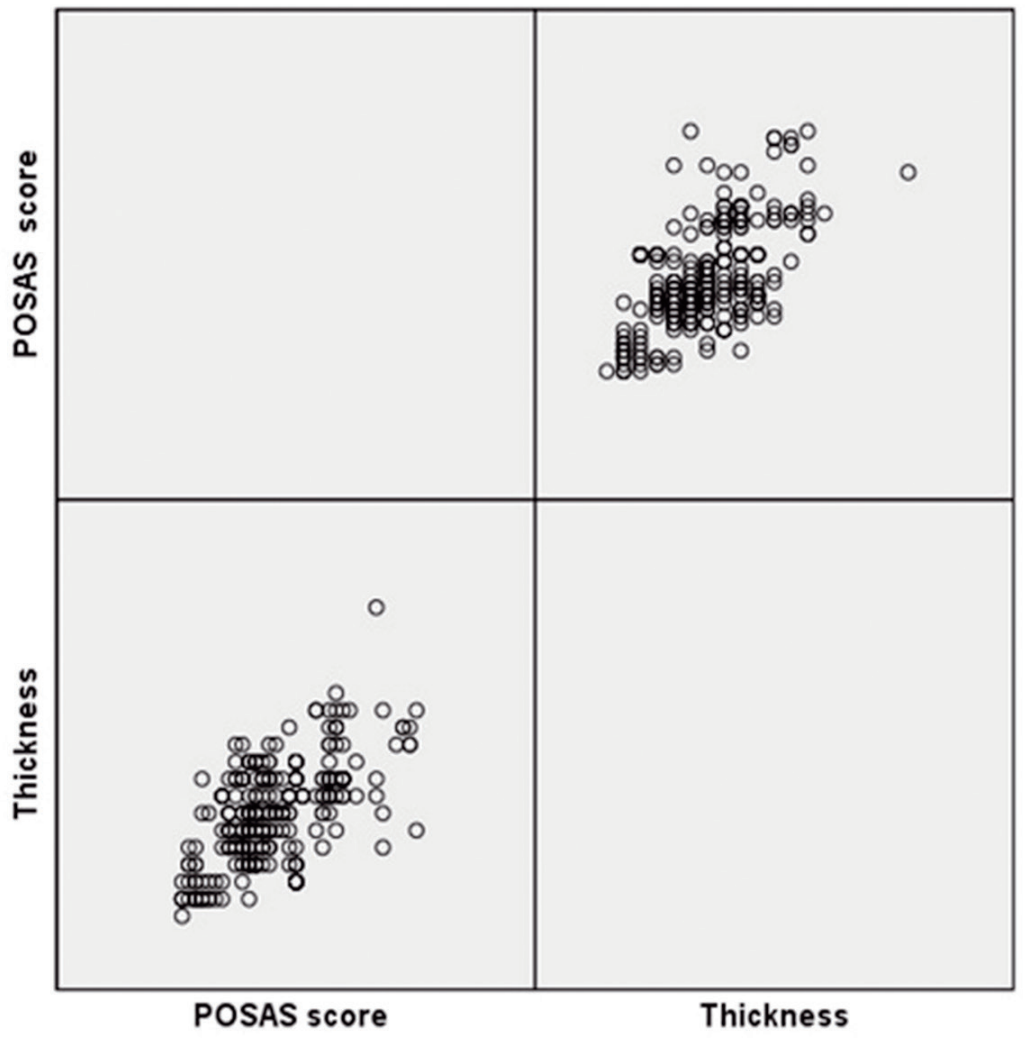

Fig. 4. Scatter plot of all cases. The partial correlation coefficient between score and thickness is 0.596 . Correlation is significant at the 0.01 level. POSAS, Patient and Observer Scar Assessment Scale.

poorer aesthetic results. Factors affecting scar formation such as skin or wound tension, scars that cross joints or skin creases, age, genetic factors, and endocrine factors are well known $[2,9,10]$. But little has been reported about the patient-specific factors such as effect of dermal thickness. We reviewed several studies on the association between dermal thickness and scar formation and planned a study to objectively confirm the findings. Ince et al. [4] studied the association between dermal thickness and scar formation after breast reduction surgery and found that a thick dermis could be a risk factor for lateral scar formation. This corresponds with our results. However, according to a study by Verim et al. [5], the skin type and dermal thickness had no association with hypertrophic scarring.

We also noted that elderly patients had lesser hypertrophic scar formation than young patients [11]. As people age, dermal thickness [12] and skin collagen density decrease [11,13], in association with changes in body fat, sunlight exposure, and hydroxyvitamin D levels. Our study showed that the incidence of hypertrophic 
scar formation increased as dermal thickness increased in areas with the same skin tension. Therefore, it is thought that dermal thinning in elderly patients, in addition to decreased skin tension with aging, may be another factor associated with decreased hypertrophic scar formation.

The mechanism by which dermal thickness affects scar formation is unclear. We surmise that collagen distribution in the dermal layer may be a factor that influences scar formation. While collagen fiber type I is distributed throughout the entire dermal layer, type III fiber is only distributed in the papillary and subpapillary layers [14]. Reticular dermis accounts for most of the dermis [15]. It is known that type I fiber is mainly distributed in the reticular dermis [16]. In a patient with thicker dermis, the component ratio of type I collagen is expected to be higher than that of type III collagen. The mechanism remains unclear, but the large role of type III collagen in scarless fetal wound healing is already known [17]. Thus, a higher proportion of collagen type I than type III could have an effect on poor scar formation and hypertrophic scarring. Microscopic evaluation of collagen architecture in patients with thick dermis might help in accurate prediction of scar formation. Further studies are needed to confirm this finding.

Even with exclusion of hypertrophic scars, the score was high. The scar score includes not only hypertrophic scars, but also depressed scars and stretched scars. Therefore, a thick dermis can increase the probability of hypertrophic scar formation, and can also result in depressed and stretched scars.

Dermal thickness can differ between men and women, but can also differ depending on the area of the body [18]. Because many variables have an effect on scar formation [2,3,10], use of the same surgical site, incision, surgeon, wound dressings, operative duration, follow-up period, and scar management greatly supports the validity of our study. In addition, we excluded patient-specific factors such as radiation therapy, steroid injection, and high body mass index, which can affect scar formation [2].

It has been reported that advising patients about risk factors and lowering expectations prior to thyroidectomy had a positive effect on patient satisfaction. Identification of a group at high risk for scar formation in advance of surgery, combined with patient education, can contribute to increased patient satisfaction. In ad- dition, identifying factors predicting hypertrophic scar and keloid development before surgery may predict the need for preoperative intralesional steroid injection or laser therapy, as well as radiation therapy, thereby inducing better scar formation [19-21].

There are limitations to this study. It is more difficult to accurately measure dermal thickness with ultrasonography than with microscopy after biopsy, and ultrasonography is also error-prone. Ince et al. [4] used biopsies to measure dermal thickness, and reported results different from those of Verim et al. [5]. However, the authors [5] did not perform biopsies and instead obtained dermis from the nasal columella, which is very thin and is thus errorprone. As the thyroidectomy method we used left the skin intact, we were unable to perform biopsies. However, a future study with a new design and use of skin biopsies would yield more accurate results. As mentioned earlier, despite the potential for errors, ultrasound measurement offers the advantage of being noninvasive, and can be used at any place on the body at lower cost [22]. We were unable to measure features distinguishing between epidermis and dermis due to the limitations of ultrasonography. A study designed to separately examine the effects of dermis and epidermis on scar formation may be useful because it is thought that these separately influence scar formation.

Dermal thickness in the anterior neck region was found to be a risk factor for hypertrophic scarring. In addition, as dermal thickness increases, the more negative effect on scar formation. Thus, use of ultrasonography to measure dermal thickness before surgery may not only aid in prediction of risk for hypertrophic scarring and poor scar formation, but may also aid in postoperative scar management, with increased patient satisfaction.

\section{CONFLICT OF INTEREST}

No potential conflict of interest relevant to this article was reported.

\section{PATIENT CONSENT}

The patients provided written informed consent for the publication and the use of their images. 


\section{REFERENCES}

1. Kim HY, Kim JW, Park JH, Kim JH, Han YS. Personal factors that affect the satisfaction of female patients undergoing esthetic suture after typical thyroidectomy. Arch Plast Surg 2013;40:414-24.

2. Lorenz P, Bari AS. Scar prevention, treatment, and revision. In: Neligan PC, editor. Plastic surgery. 3rd ed. London: Elsevier Saunders; 2012.p. 297-318.

3. Broughton G 2nd, Janis JE, Attinger CE. Wound healing: an overview. Plast Reconstr Surg 2006;117(7 Suppl):le-S-32e-S.

4. Ince B, Dadaci M, Oltulu P, Altuntas Z, Bilgen F. Effect of dermal thickness on scars in women with type III-IV fitzpatrick skin. Aesthetic Plast Surg 2015;39:318-24.

5. Verim A, Duymus R, Calim OF, Karaca CT, Ozkul MH, Yasar H, et al. Effect of nose skin on the columellar incision scar in a Turkish population. Otolaryngol Head Neck Surg 2013;149:438-44.

6. Draaijers LJ, Tempelman FR, Botman YA, Tuinebreijer WE, Middelkoop E, Kreis RW, et al. The patient and observer scar assessment scale: a reliable and feasible tool for scar evaluation. Plast Reconstr Surg 2004;113:1960-5.

7. van de Kar AL, Corion LU, Smeulders MJ, Draaijers LJ, van der Horst CM, van Zuijlen PP. Reliable and feasible evaluation of linear scars by the Patient and Observer Scar Assessment Scale. Plast Reconstr Surg 2005;116:514-22.

8. Perry DM, McGrouther DA, Bayat A. Current tools for noninvasive objective assessment of skin scars. Plast Reconstr Surg 2010;126:91223.

9. Wolfram D, Tzankov A, Pulzl P, Piza-Katzer H. Hypertrophic scars and keloids: a review of their pathophysiology, risk factors, and therapeutic management. Dermatol Surg 2009;35:171-81.

10. Kim JH, Sung JY, Kim YH, Lee YS, Chang HS, Park CS, et al. Risk fac- tors for hypertrophic surgical scar development after thyroidectomy. Wound Repair Regen 2012;20:304-10.

11. Gosain A, DiPietro LA. Aging and wound healing. World J Surg 2004;28:321-6.

12. Need AG, Morris HA, Horowitz M, Nordin C. Effects of skin thickness, age, body fat, and sunlight on serum 25 -hydroxyvitamin D. Am JClin Nutr 1993;58:882-5.

13. Shuster S, Black MM, McVitie E. The influence of age and sex on skin thickness, skin collagen and density. Br J Dermatol 1975;93:639-43

14. Shimizu H. Structure and function of the skin. In: Shimizu H, editor. Shimizu's textbook of dermatology. Sapporo: Hokkaido University Press; 2007.p. 12-6.

15. Scherer-Pietramaggiori SS, Pietramaggiori G, Dennis P. Skin graft. In: Neligan PC, editor. Plastic surgery. 3rd ed. London: Elsevier Saunders; 2012.p. 319-38.

16. Meigel WN, Gay S, Weber L. Dermal architecture and collagen type distribution. Arch Dermatol Res 1977;259:1-10.

17. Whitby DJ, Ferguson MW. The extracellular matrix of lip wounds in fetal, neonatal and adult mice. Development 1991;112:651-68.

18. Lee Y, Hwang K. Skin thickness of Korean adults. Surg Radiol Anat 2002;24:183-9.

19. Tang YW. Intra- and postoperative steroid injections for keloids and hypertrophic scars. Br J Plast Surg 1992;45:371-3.

20. Henderson DL, Cromwell TA, Mes LG. Argon and carbon dioxide laser treatment of hypertrophic and keloid scars. Lasers Surg Med 1984;3:271-7.

21. Kim K, Son D, Kim J. Radiation therapy following total keloidectomy: a retrospective study over 11 years. Arch Plast Surg 2015;42:588-95.

22. Alexander H, Miller DL. Determining skin thickness with pulsed ultra sound. J Invest Dermatol 1979;72:17-9. 